\title{
Dark Matter Annihilation and Decay Searches with the High Altitude Water Cherenkov (HAWC) Observatory
}

\author{
J. Patrick Harding ${ }^{a}$ and Brenda Dingus ${ }^{* a}$ for the HAWC Collaboration ${ }^{b}$ \\ ${ }^{a}$ Los Alamos National Laboratory, Los Alamos, NM, USA \\ ${ }^{b}$ For a complete author list, see www.hawc-observatory.org/collaboration/icrc2015.php. \\ Email: jphardingelanl.gov, dinguselanl.gov
}

\begin{abstract}
In order to observe annihilation and decay of dark matter, several types of potential sources should be considered. Some sources, such as dwarf galaxies, are expected to have very low astrophysical backgrounds but fairly small dark matter densities. Other sources, like the Galactic center, are expected to have larger densities of dark matter but also have more complicated backgrounds from other astrophysical sources. To search for signatures of dark matter, the large field-of-view of the HAWC detector, covering 2 sr at a time, especially enables searches from sources of dark matter annihilation and decay, which are extended over several degrees on the sky. With a sensitivity over $2 / 3$ of the sky, HAWC has the ability to probe a large fraction of the sky for the signals of TeV-mass dark matter. In particular, HAWC should be the most sensitive experiment to signals coming from dark matter with masses greater than 10-100 TeV. We present the HAWC sensitivity to annihilating and decaying dark matter signals for several likely sources of these signals.
\end{abstract}

The 34th International Cosmic Ray Conference,

30 July- 6 August, 2015

The Hague, The Netherlands

${ }^{*}$ Speaker. 


\section{Introduction}

In recent years, the idea that dark matter is made up of weakly interacting massive particles (WIMPs) has been particularly studied (see ref. [1] for more details). In this proceeding, we consider the ability of the High Altitude Water Cherenkov (HAWC) observatory to detect gamma rays associated with annihilation and decay of multi-TeV-mass WIMP dark matter [2]. When dark matter annihilates or decays, the emitted gamma-ray spectrum is expected to peak at roughly an order-of-magnitude below the WIMP mass. This corresponds to a HAWC sensitivity to WIMPs with masses between $1 \mathrm{TeV}$ and $1 \mathrm{PeV}$. Many dark matter gamma-ray sources are expected to have large angular extent, so the HAWC Observatory is particularly sensitive to these sources. Also, because HAWC will search over 2/3 of the sky, it will be able to cross-correlate signals from many different types of dark matter sources to improve its sensitivity and decrease systematic uncertainties in the sources.

\section{Gamma Rays from Annihilating and Decaying WIMPs}

\subsection{Dark Matter Density Profiles}

From dark matter simulations, the density profile of dark matter $\rho(r)$ falls off roughly as $r^{-2}$ away from the center of a dark matter halo. Typically, the shape of this profile is chosen as either an Navarro-Frenk-White (NFW) profile [3] or an Einasto profile [4, 5], which have a central cusp for small radii. The form we use for these profiles is given in refs. [2, 6].

Other profiles which do not peak strongly toward the center of the source are also possible [7]. However, even for dark matter profiles which peak strongly toward the center of the source, the dark matter halo for most sources is large. Therefore, most dark matter signals are expected to give extended emission across the sky. For the dark matter halo parameters of each source, we use the parameters given in ref. [2].

\subsection{Gamma-ray Flux from Dark Matter Annihilation}

The gamma-ray flux from dark matter annihilation is a function of the astrophysics of the source, including the dark matter mass-density profile, $\rho(r)$, of the source as well the distance from the source to the detector, $R$, and the angle it subtends on the sky, $\Delta \Omega$. It also depends on the particle physics of the dark matter model, including the WIMP mass $M_{\chi}$, the dark matter annihilation rate $\left\langle\sigma_{\mathrm{A}} v\right\rangle$, and the spectrum $\mathrm{d} N_{\gamma} / \mathrm{d} E$ of gamma rays from each WIMP annihilation.

For dark matter annihilation, the gamma-ray flux depends on the square of the dark matter density $\rho$ as

$$
\frac{\mathrm{d} F}{\mathrm{~d} E}_{\text {annihilation }}=\frac{\left\langle\sigma_{\mathrm{A}} v\right\rangle}{8 \pi M_{\chi}^{2}} \frac{\mathrm{d} N_{\gamma}}{\mathrm{d} E} \int_{\Delta \Omega} \mathrm{d} \Omega \int \mathrm{d} x \rho^{2}\left(r_{\mathrm{gal}}(\theta, x)\right)
$$

To account for the total observed dark matter in the source, this flux is integrated over the line-ofsight distance $x$, where the distance from the center of the source is given by

$$
r_{\mathrm{gal}}(\theta, x)=\sqrt{R^{2}-2 x R \cos (\theta)+x^{2}}
$$


for an angle $\theta$ between the line-of-sight and the direction to the source center. The gamma-ray spectrum $d N_{\gamma} / d E$ depends both on the species of particle which the dark matter annihilates into and the dark matter mass.

\subsubsection{Dark Matter Substructure}

Although the average shape of the dark matter density follows the profiles described in section 2.1, dark matter simulations show that dark matter halos also contain many smaller subhalos, local overdensities of dark matter. Because the gamma-ray flux from dark matter annihilation goes as the square of the dark matter density, these denser regions actually contribute most of the flux. This is particularly important for more massive objects, such as galaxies and galaxy clusters, which have more mass and therefore more massive and denser subhalos. Here, we consider the substructure model of ref. [8]. In this model, the dark matter flux is "boosted" by $\sim 15$ for the M31 galaxy and by $\sim 35$ for the Virgo cluster due to these subhalos. In figures 2 and 3, we show the limits both with and without this enhancement from substructure, for comparison. Additionally, note that these values are very conservative; in the substructure model of ref. [9], for example, the Virgo cluster can have its flux increased by 1000 times over the flux of the smooth profile.

\subsubsection{Dark Matter Annihilation Cross-section}

Due to the uncertainty of the particle nature of dark matter, many different models for the dark matter annihilation cross-section have been hypothesized (for a review, see [1]). However, one of the most common models is a dark matter particle which is produced thermally in the early universe. If such a particle annihilated in the early universe, then the strength of the dark matter annihilation cross-section should determine the amount of relic dark matter observed today. Dark matter produced through such a mechanism should have a cross-section between $\left\langle\sigma_{\mathrm{A}} v\right\rangle \approx 2.2 \times$ $10^{-26} \mathrm{~cm}^{3} \mathrm{~s}^{-1}$ and $\left\langle\sigma_{\mathrm{A}} v\right\rangle \approx 4.4 \times 10^{-26} \mathrm{~cm}^{3} \mathrm{~s}^{-1}$, depending on the nature of the dark matter [10]. The cross-section in the thermal dark matter model is largely independent of the dark matter mass and is typically given as $\left\langle\sigma_{\mathrm{A}} v\right\rangle \approx 3 \times 10^{-26} \mathrm{~cm}^{3} \mathrm{~s}^{-1}$, which is the value we show in this paper.

Another common model consists of dark matter which interacts with light dark-sector gauge bosons. In this model, called Sommerfeld enhancement [11], the exchange of these light bosons can create a resonance between the dark matter particles, increasing their cross-section by several orders of magnitude. The cross-section in models with Sommerfeld enhancement is dependent on the relative velocity of the dark matter particles as well as the mass and coupling of the dark matter to the light bosons. For the high dark matter masses considered in this paper, however, even standard model $W$ and $Z$ bosons are light enough to give a Sommerfeld enhancement effect. Any dark matter which is much heavier than the $W$-mass and couples to standard model gauge bosons should produce this effect. For the WIMP model we consider here, we assume a weakscale coupling between the dark matter and the $W$ boson, using the formalism of ref. [12].

\subsection{Gamma-ray Flux from Dark Matter Decay}

In order for dark matter to survive with the present observed density, dark matter particles must be fairly stable. However, it is possible that dark matter, while long-lived compared to the age of the universe, may have a finite lifetime. Even if the dark matter lifetime were much longer than 
the age of the universe, the astrophysical effects of the decay would be observable. Observations of astrophysical signals, such as the neutrino flux observed by the IceCube detector, could even be the first observations of PeV-mass dark matter decays $[13,14,15,16]$.

The gamma-ray flux from dark matter decay is similar to the flux from annihilating dark matter (equation 2.1). The flux for decay depends on the dark matter decay lifetime $\tau_{\chi}$ instead of the annihilation cross-section and the gamma-ray spectrum for each dark matter decay. Also, the flux depends on a single power of the dark matter density $\rho$ as

$$
\frac{\mathrm{d} F}{\mathrm{~d} E} \text { decay }_{4 \pi \tau_{\chi} M_{\chi}} \frac{\mathrm{d} N_{\gamma}}{\mathrm{d} E} \int_{\Delta \Omega} \mathrm{d} \Omega \int \mathrm{d} x \rho\left(r_{\mathrm{gal}}(\theta, x)\right) .
$$

Because the flux is linearly proportional to the dark matter density, dark matter substructures do not appreciably affect the flux from dark matter decay. Also, decaying dark matter also gives more spatially-extended emission than annihilation. The flux from decay is much less sensitive to the shape of the dark matter density profile than in annihilation. Therefore, the best limits on dark matter decay are expected to come from the most massive objects in the universe, including galaxies and galaxy clusters. Because the flux for dark matter decay is inversely proportional to the dark matter lifetime $\tau_{\chi}$, the limits on the dark matter lifetime are lower limits, rather than the upper limits which are calculated for dark matter annihilation.

\subsection{Gamma-ray Spectrum from Dark Matter Annihilation and Decay}

Typically, the gamma-ray spectra are calculated assuming a 100 percent branching fraction of the dark matter into a single final state. The particles in this final state then decay to stable particles close to the dark matter source, which produces gamma rays. For dark matter annihilation, the maximum energy of a produced photon is the dark matter mass, while for dark matter decay the maximum photon energy is half the dark matter mass. Additionally, the expected dark matter gamma-ray spectra from annihilation and decay are different from typical astrophysical spectra. The gamma-ray spectra for $10 \mathrm{TeV}$ dark matter particles annihilating into bottom quarks, tau leptons, and $W$ bosons are shown in figure 1.

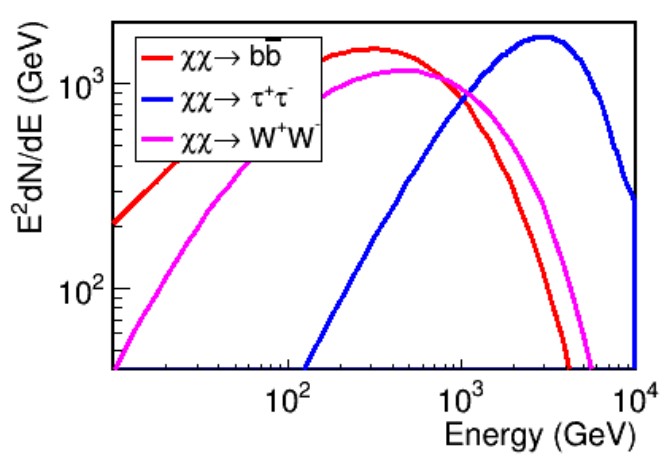

Figure 1: The gamma-ray spectra for $10 \mathrm{TeV}$ dark matter particles annihilating into bottom quarks (red), tau leptons (blue), and $W$ bosons (magenta). The spectrum for the leptons is harder than for the quarks and bosons. All spectra cut off sharply at the dark matter mass.

To calculate the dark matter gamma-ray spectra presented here, we use the PYTHIA 6.4 code [17]. This simulates radiation from charged particles as well as the production of gammas in decays of particles such as the $\pi^{0}$. We run this program following the methods of ref. [18] to calculate the average spectrum $\mathrm{d} N_{\gamma} / \mathrm{d} E$ of gamma rays from each WIMP annihilation or decay. 

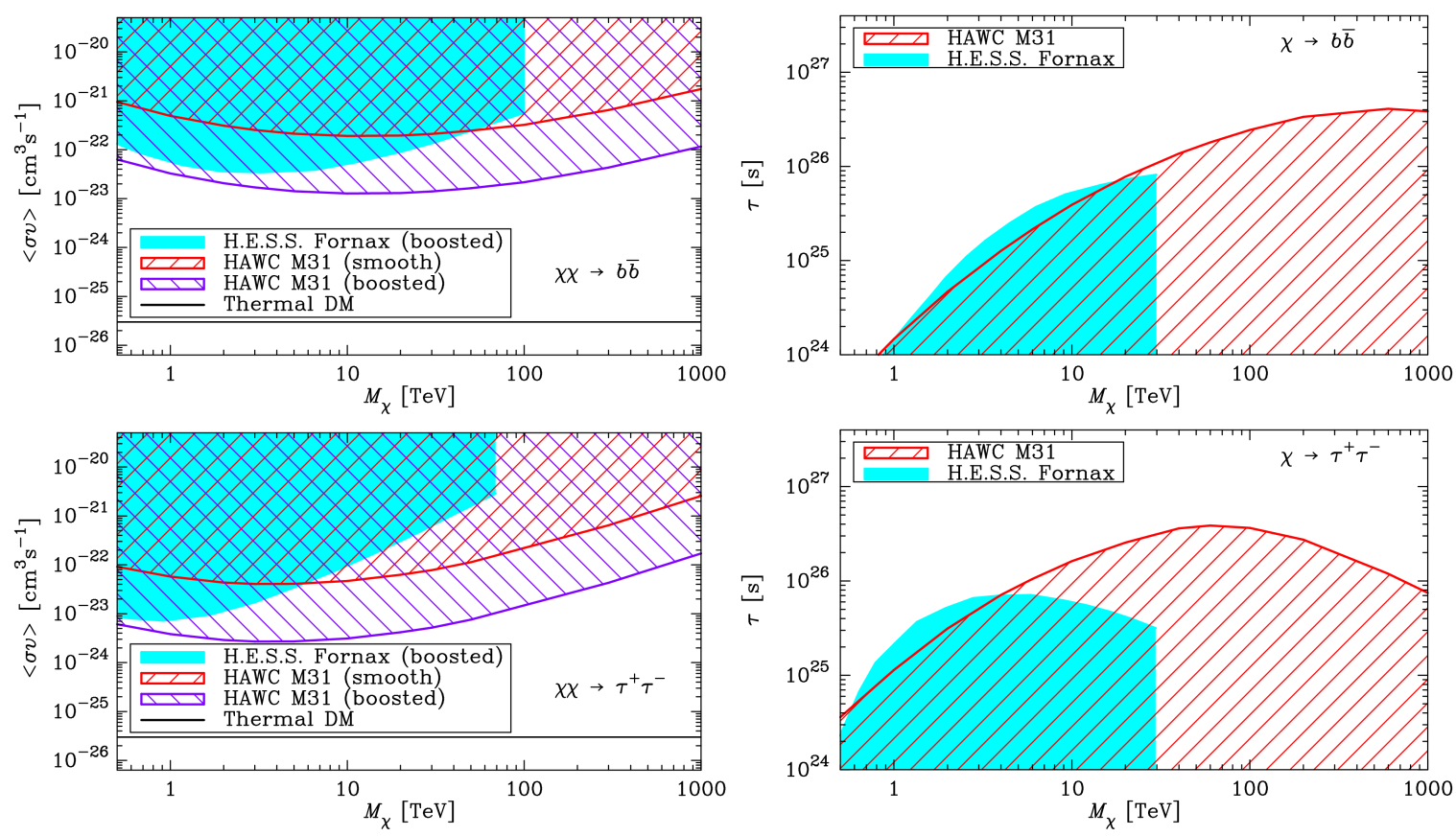

Figure 2: The projected dark matter limits from M31 for HAWC after five years, as a function of the dark matter particle mass. The plots on the left are the expected HAWC upper limits on the dark matter crosssection $\left\langle\sigma_{\mathrm{A}} v\right\rangle$ for dark matter which annihilates into bottom quarks (top) and tau leptons (bottom). The figures on the right are the expected HAWC lower limits on the dark matter lifetime $\tau_{\chi}$ for dark matter which decays into bottom quarks (top) and tau leptons (bottom). In the annihilation plots, the limits are shown both for just the smooth dark matter halo (red hatched) or with a substructure boost from ref. [19] (purple hatched). For comparison, the cyan regions show the measured limit from the H.E.S.S. observatory's observations of the Fornax cluster [19, 20], both for annihilating dark matter boosted using the substructure boost model of ref. [8] and for decaying dark matter, for comparison. The black line in the annihilation plots shows the expected cross-section for thermally-produced WIMP dark matter. All limits are at 95\% CL.

\section{HAWC Sensitivity to Dark Matter Annihilation and Decay}

Using the HAWC simulation [21, 2], we determined the 5-year HAWC sensitivity to dark matter annihilation and decay for several dark matter annihilation channels and a range of dark matter masses. In figure 2, we show the HAWC sensitivity to dark matter annihilation and decay in the M31 galaxy. For comparison, we also show the measured limit from the H.E.S.S. observatory's observations of the Fornax cluster [19, 20], which is one of the strongest dark matter limits from galaxy clusters in the $\mathrm{TeV}$ mass range. In the annihilation figures, the limit is shown both for just the smooth dark matter density profile and for a substructure boost factor from the model of ref. [8]. The H.E.S.S. Fornax limits have an included substructure boost factor from the ref. [8] model. For TeV-mass dark matter annihilation, the limits from M31 should give HAWC's strongest limits. For decaying dark matter, the HAWC limits will place the strongest limits from a TeV experiment.

In figure 3, we show similar limits for the Virgo cluster. The Virgo cluster limits from HAWC on dark matter annihilation should be similar to the H.E.S.S. Fornax limit below $6 \mathrm{TeV}(2 \mathrm{TeV})$ for the hadronic (leptonic) annihilation channel, with the HAWC limits much more constraining at 

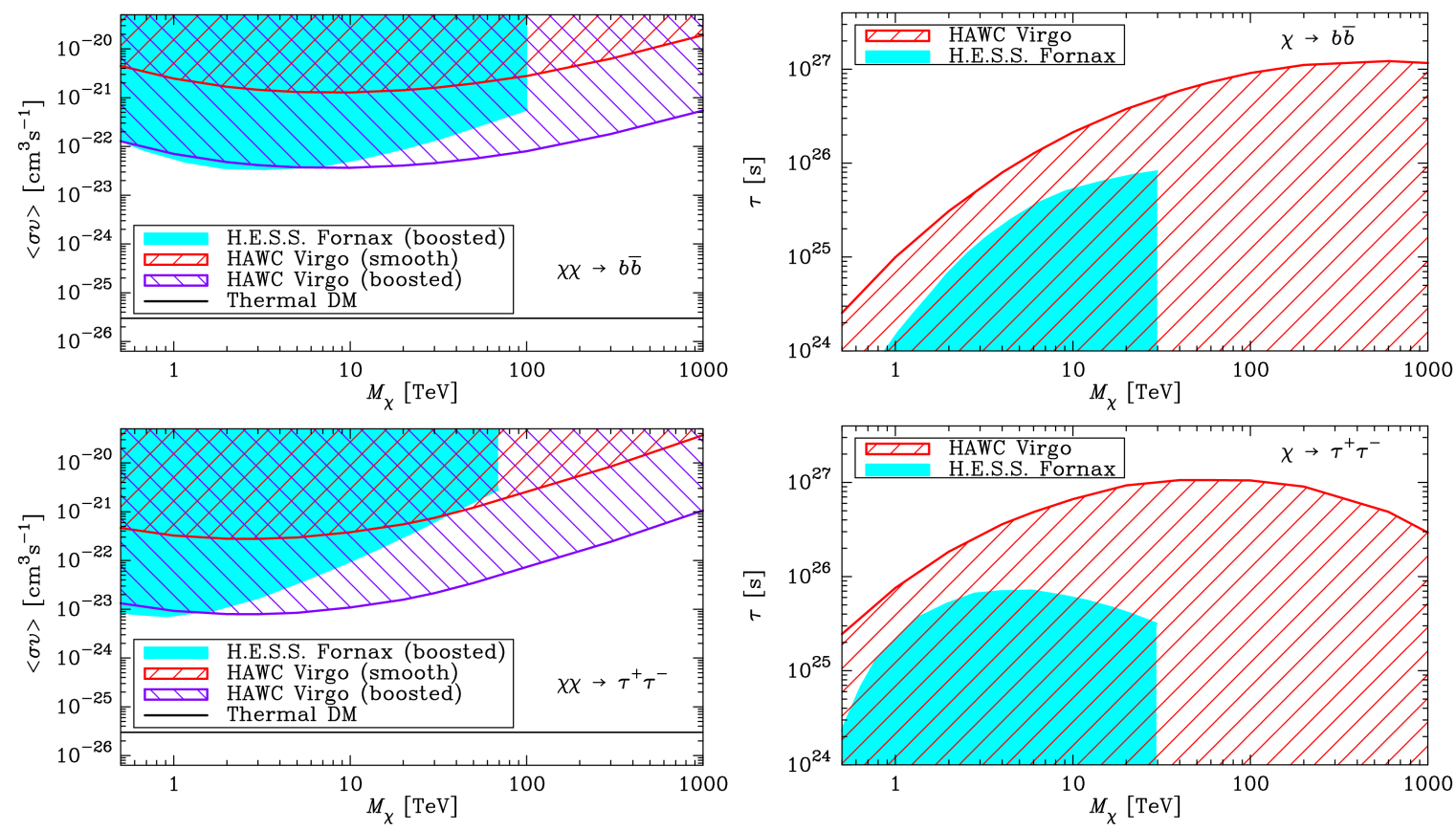

Figure 3: The projected dark matter limits from the Virgo Cluster for HAWC after five years, as a function of the dark matter particle mass. The figures follow the same format as those in figure 2.

higher masses. Because the Virgo cluster is the most massive dark matter object considered in this study, the Virgo cluster will give the strongest HAWC limit on decaying dark matter.

Figure 4 shows the relative HAWC sensitivity to annihilating dark matter for several sources and annihilation channels. Although the substructure enhancement for the Virgo cluster is larger than for M31, the annihilation limit from M31 will be somewhat stronger than from the Virgo cluster. At the largest masses, however, the Galactic center may provide the strongest limits from HAWC. This is because the Galactic center peaks at $48^{\circ}$ from HAWC zenith, so many of the low-energy photons from the Galactic center dark matter range out in the atmosphere and are not observable by HAWC. The possibilities that contamination from the Galactic plane or M87 could worsen these limits has been considered, but is expected to affect the limits by less than $32 \%$ [2]. The purple lines show the expected cross-section for the thermal dark matter model. In the $W^{+} W^{-}$ channel plot, we also show the expected cross-section for a Sommerfeld-enhanced dark matter model. For this model, HAWC should be able to rule out a range of dark matter masses after 5 years of observations.

\section{Conclusions}

With 5 years of observations, HAWC will be one of the most sensitive experiments to TeVmass dark matter annihilation and decay [2]. With its sensitivity to high energies, HAWC will be able to observe signatures of dark matter with masses above $1 \mathrm{TeV}$. Especially for extended dark matter sources, including large sources such as M31 and the Virgo cluster, HAWC's large fieldof-view will enable observations of much of the dark matter halo. The HAWC observations of 

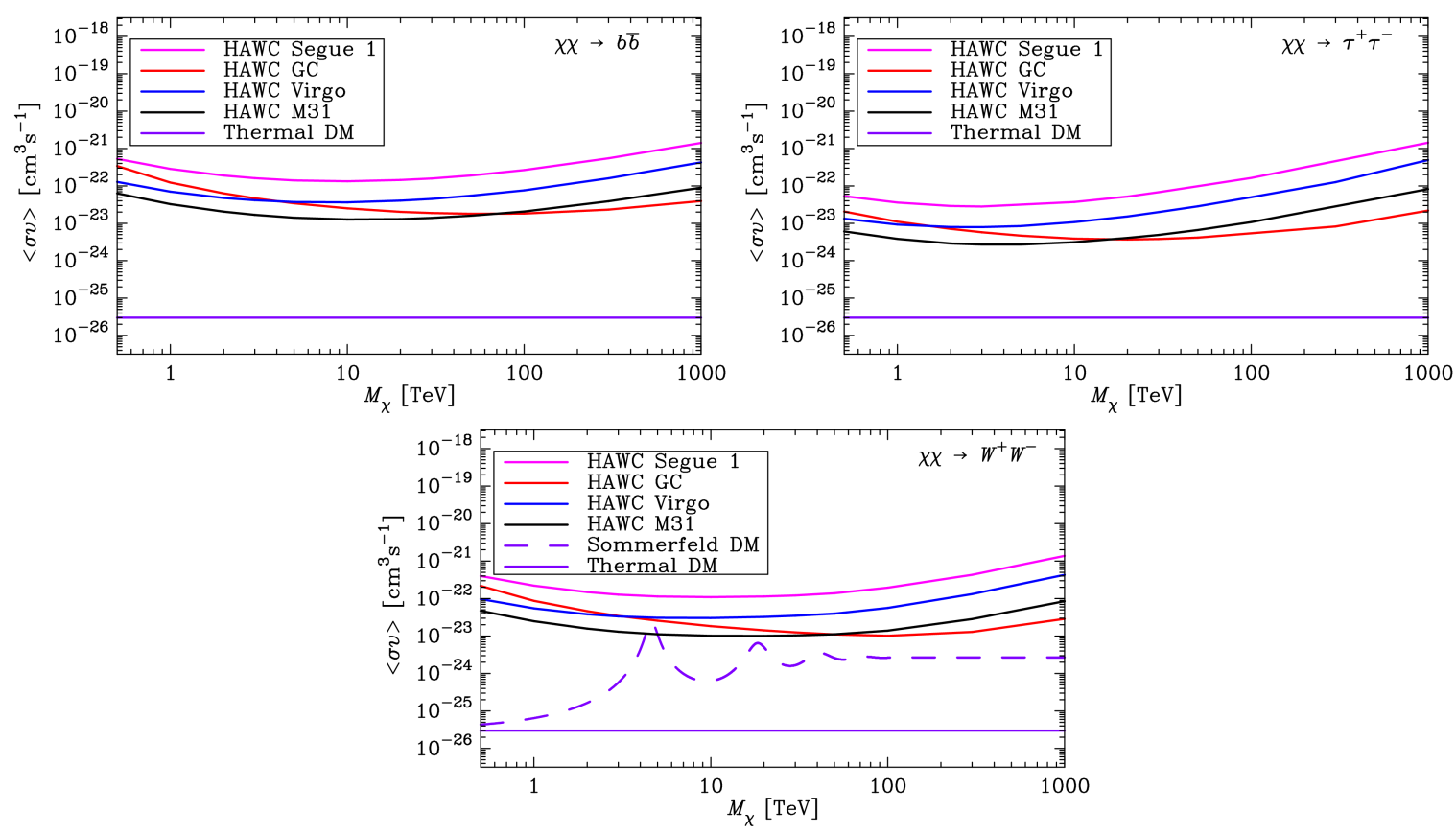

Figure 4: The projected dark matter upper limits from several sources for HAWC after five years, for the $b \bar{b}, \tau^{+} \tau^{-}$, and $W^{+} W^{-}$dark matter annihilation channels. The curves are for the Segue 1 dwarf galaxy (magenta), the Milky Way Galactic center (red), the Virgo cluster with substructure boost (blue), and the M31 galaxy with substructure boost (black). The substructure boosts follow the model of ref. [8]. The solid purple line shows the expected cross-section for thermally-produced WIMP dark matter. In the $W^{+} W^{-}$ figure, the dashed purple line indicates the cross-section expected for dark matter with natural Sommerfeld enhancement. All limits are at 95\% CL.

the M31 galaxy, with its expected substructure, will place the best HAWC constraint on models of dark matter annihilation. For decaying dark matter, HAWC observations of the Virgo cluster will provide the best $\mathrm{TeV}$-scale limits on the dark matter lifetime. Overall, the HAWC observatory promises to open a new window on searches for dark matter annihilation and decay.

\section{Acknowledgments}

We acknowledge the support from: the US National Science Foundation (NSF); the US Department of Energy Office of High-Energy Physics; the Laboratory Directed Research and Development (LDRD) program of Los Alamos National Laboratory; Consejo Nacional de Ciencia y Tecnología (CONACyT), Mexico (grants 260378, 55155, 105666, 122331, 132197, 167281, 167733); Red de Física de Altas Energías, Mexico; DGAPA-UNAM (grants IG100414-3, IN108713, IN121309, IN115409, IN111315); VIEP-BUAP (grant 161-EXC-2011); the University of Wisconsin Alumni Research Foundation; the Institute of Geophysics, Planetary Physics, and Signatures at Los Alamos National Laboratory; the Luc Binette Foundation UNAM Postdoctoral Fellowship program.

\section{References}

[1] J. L. Feng, Dark Matter Candidates from Particle Physics and Methods of Detection, Ann.Rev.Astron.Astrophys. 48 (2010) 495, [arXiv: 1003.0904 ]. 
[2] HAWC Collaboration, A. Abeysekara et al., Sensitivity of HAWC to high-mass dark matter annihilations, Phys.Rev. D90 (2014), no. 12 122002, [arXiv: 1405.1730 ].

[3] J. F. Navarro, C. S. Frenk, and S. D. White, A Universal density profile from hierarchical clustering, Astrophys.J. 490 (1997) 493-508, [astro-ph/9611107].

[4] J. Stadel et al., Quantifying the heart of darkness with GHALO - a multi-billion particle simulation of our galactic halo, Mon. Not. Roy. Astron. Soc. 398 (2009) L21, [arXiv: 0808 . 2981].

[5] J. F. Navarro et al., The Diversity and Similarity of Cold Dark Matter Halos, arXiv : 0810.1522.

[6] HAWC Collaboration, M. L. Proper, B. Dingus, and J. P. Harding, First Limits on the Dark Matter Cross-Section with the HAWC Observatory, in Proc. 34th ICRC, (The Hague, The Netherlands), August, 2015.

[7] A. Burkert, The Structure of dark matter halos in dwarf galaxies, IAU Symp. 171 (1996) 175, [astro-ph/9504041].

[8] M. A. Sanchez-Conde and F. Prada, The flattening of the concentration-mass relation towards low halo masses and its implications for the annihilation signal boost, Mon.Not.Roy.Astron.Soc. 442 (2014), no. 3 2271-2277, [arXiv:1312.1729].

[9] J. Han et al., Constraining Extended Gamma-ray Emission from Galaxy Clusters, Mon.Not.Roy.Astron.Soc. 427 (2012) 1651-1665, [arXiv: 1207.6749 ].

[10] G. Steigman, B. Dasgupta, and J. F. Beacom, Precise Relic WIMP Abundance and its Impact on Searches for Dark Matter Annihilation, Phys.Rev. D86 (2012) 023506, [arXiv: 1204 . 3622].

[11] M. Lattanzi and J. I. Silk, Can the WIMP annihilation boost factor be boosted by the Sommerfeld enhancement?, Phys.Rev. D79 (2009) 083523, [arXiv: 0812.0360].

[12] J. L. Feng, M. Kaplinghat, and H.-B. Yu, Sommerfeld Enhancements for Thermal Relic Dark Matter, Phys.Rev. D82 (2010) 083525, [arXiv: 1005 .4678].

[13] IceCube Collaboration, M. Aartsen et al., First observation of PeV-energy neutrinos with IceCube, Phys.Rev.Lett. 111 (2013) 021103, [arXiv: 1304.5356$].$

[14] IceCube Collaboration, M. Aartsen et al., Evidence for High-Energy Extraterrestrial Neutrinos at the IceCube Detector, Science 342 (2013), no. 6161 1242856, [arXiv: 1311. 5238].

[15] A. Esmaili and P. D. Serpico, Are IceCube neutrinos unveiling PeV-scale decaying dark matter?, JCAP 1311 (2013) 054, [arXiv: 1308 .1105].

[16] Y. Bai, R. Lu, and J. Salvado, Geometric Compatibility of IceCube TeV-PeV Neutrino Excess and its Galactic Dark Matter Origin, arXiv: 1311.5864.

[17] T. Sjostrand, S. Mrenna, and P. Z. Skands, PYTHIA 6.4 Physics and Manual, JHEP 0605 (2006) 026, [hep-ph/0603175].

[18] K. N. Abazajian and J. Harding, Constraints on WIMP and Sommerfeld-Enhanced Dark Matter Annihilation from HESS Observations of the Galactic Center, JCAP 1201 (2012) 041, [arXiv:1110.6151].

[19] A. Abramowski et al., Search for Dark Matter Annihilation Signals from the Fornax Galaxy Cluster with H.E.S.S, Astrophys.J. 750 (2012) 123, [arXiv: 1202.5494 ].

[20] M. Cirelli, E. Moulin, P. Panci, P. D. Serpico, and A. Viana, Gamma ray constraints on decaying dark matter, Phys. Rev. D 86 (Oct., 2012) 083506, [arXiv: 1205.5283 ].

[21] A. Abeysekara et al., Sensitivity of the High Altitude Water Cherenkov Detector to Sources of Multi-TeV Gamma Rays, Astropart.Phys. 50-52 (2013) 26-32, [arXiv: 1306.5800 ]. 